\title{
Statistical approaches for analysis of mapping quality for single-cell sequencing data
}

\author{
I.I. Abnizova ${ }^{1,2 *}$, R. te Boekhorst ${ }^{3}$, N. Beka ${ }^{3}$, F.M. Naumenko ${ }^{4}$, A.V. Tsukanov ${ }^{4,5}$, \\ Y.L. Orlov ${ }^{4,5,6 *}$ \\ ${ }^{1}$ Wellcome Trust Sanger Institute, Cambridge, UK \\ ${ }^{2}$ Babraham Institute, Cambridge, UK \\ ${ }^{3}$ University of Hertfordshire, Hertfordshire, UK \\ ${ }^{4}$ Novosibirsk State University, Novosibirsk, Russia \\ ${ }^{5}$ Institute of Cytology and Genetics SB RAS, Novosibirsk, Russia \\ ${ }^{6}$ Institute of Marine Biology Researches of the RAS, Sevastopol, Russia \\ *e-mail:irina_abnizova@yahoo.co.uk;orlov@bionet.nsc.ru
}

Key words: Next-generation sequencing, DNA alignment, read density distribution.

Motivation and Aim: Bioinformatics analysis is essential in providing biological insights for single-cell experiments, such as detecting variants, quantifying gene expression, and subpopulation detection. However, conventional tools developed for bulk-cell genomics cannot be directly applied to single-cell sequencing data [1].

Methods and Algorithms: This low coverage characteristic of single-cell sequencing data has posed difficulties in the variant calling procedure. Most bioinformatics tools employ sequence read density to call variants [2]. Single nucleotide polymorphisms and small insertions/deletions with low read support are excluded in conventional bioinformatics tools. In genome assemblies, the low coverage and heterogeneity of single-cell sequencing data also bring substantial disadvantages, leading to truncated sequences with high numbers of sequencing artefacts. Recently, single-cell assemblers such as SPAdes and IDBA-UD have been specifically developed to overcome the challenge of amplification artefacts in single-cell sequencing and generate more precise single-cell genomic assemblies. Common gene expression metrics such as Fragments Per Kilobase Million/Reads Per Kilobase Million (FPKM/RPKM) do not address these 3 '-end biases and thus have a limited application for scRNA sequencing. Using own scripts we investigated chromosome mapping quality and possible artefacts [3].

Results and conclusion: We applied or approaches to study Differentially Chromatin accessed regions (DARs) and Diff Methylated Regions (DMR). The generation of artificial data by mapping of generated reads to a reference genome is justified from the point of view of reducing the benchmarking time. We will review current state of art of mapping programs in this research area.

Acknowledgements: The research has been supported by RFBR. Computing done at Siberian Supercomputer center SB RAS was supported by budget project No. 0324-20180017.

\section{References}

1. Yuan Y. et al. (2018) Single-Cell Genomic Analysis in Plants. Genes (Basel). 9(1):E50.

2. Valihrach L. et al. (2018) Platforms for Single-Cell Collection and Analysis. Int J Mol Sci. 19(3). pii: E807.

3. Naumenko F.M. et al. (2018) Novel read density distribution score shows possible aligner artefacts, when mapping a single chromosome. BMC Genomics. 19(Suppl 3):92. 